\title{
Pour un meilleur équilibre de la cellule familiale. Un regard sur les Self-Help Groups en Inde du Sud
}

For a Better Family Equilibrium. An exploration of the Self-Help-Groups in South India

\section{Philippe Broda}

\section{OpenEdition}

\section{Journals}

Édition électronique

URL : http://journals.openedition.org/economierurale/3462

DOI : 10.4000/economierurale.3462

ISSN : 2105-2581

\section{Éditeur}

Société Française d'Économie Rurale (SFER)

Édition imprimée

Date de publication : 30 mai 2012

Pagination : 83-97

ISSN : 0013-0559

Référence électronique

Philippe Broda, «Pour un meilleur équilibre de la cellule familiale. Un regard sur les Self-Help Groups en Inde du Sud », Économie rurale [En ligne], 329 | mai-juin 2012, mis en ligne le 30 mai 2014, consulté le 02 mai 2019. URL : http://journals.openedition.org/economierurale/3462 ; DOI : 10.4000/ economierurale.3462 


\title{
Pour un meilleur équilibre de la cellule familiale Un regard sur les Self-Help Groups en Inde du Sud
}

\author{
Philippe BRODA • Enseignant-Chercheur à NOVANCIA, Business School, Paris \\ pbroda@novancia.fr
}

A u début des années 1980, les politiques de lutte contre la pauvreté conduites par l'État avaient échoué en Inde. Les projets réformistes se sont succédés mais sans amélioration notable pour les plus démunis. Les directives de l'État central ne parvenaient pas à atteindre les villages et les bidonvilles : les intentions généreuses ne suffisaient manifestement pas. Fort de ce constat, l'État décida de changer de mode d'intervention. Il se résolut à s'appuyer sur les initiatives d'acteurs locaux. De ce point de vue, l'action de Myrada, une ONG pionnière, pouvait servir de modèle. Elle s'était mise à promouvoir le développement de « groupes de solidarité ». L'objectif était de les rendre éligibles au microcrédit afin que leurs membres puissent entreprendre des activités économiques et s'extraire ainsi de la pauvreté. La tâche de l'État a alors consisté à favoriser la systématisation de cette démarche. L'évolution de la mission de la National Bank for Agriculture and Rural Development (NABARD) est symptomatique de ce changement dans le rôle de la puissance publique. Auparavant, sur les injonctions de l'État, cette banque publique était supposée accorder des prêts aux plus défavorisés qui étaient cependant très réticents à contracter des emprunts auprès d'elle. La NABARD s'est muée en institution au service de la microfinance, travaillant en collaboration avec les ONG qui stimulent désormais la création des groupes de solidarité et les encadrent.

En Inde du Sud, ces groupes constitués principalement de femmes sont appelés SelfHelp Groups (SHG). Ils ont effectivement pour finalité d'améliorer la situation économique de familles qui vivent parfois dans un

état de dénuement extrême. Mais les SHG ne sont pas de simples pourvoyeurs de financement. Leur action incorpore également un volet social : ils occasionnent la modification de l'articulation entre le principe de liaison avec celui de conflit à l'intérieur même de la cellule familiale. Les ONG ne l'ignorent pas. Elles espèrent que le premier principe, qui sera ici nommé eros puisse provoquer un recul du second qui, lui, sera désigné sous le nom de polemos. L'empowerment des femmes doit rééquilibrer le fonctionnement de la famille. Cet article se propose d'examiner si les SHG remplissent leur mission à la fois économique et sociale.

Pour ce faire, il conviendra d'abord d'analyser le dispositif matériel d'évaluation des SHG par la NABARD, leur principal financeur, pour comprendre comment cet organisme définit et évalue les SHG, comment sont définies les «bonnes pratiques » en la matière, les objectifs assignés aux SHG, bref le référentiel dans lequel ils sont inscrits par leur promoteur, l'État indien. Et ensuite, pouvoir comparer la réalité des SHG à ce référentiel et évaluer la capacité des SHG à atteindre ces objectifs.

Faisant suite à une enquête de terrain de dix jours menée au Tamil Nadu durant l'été 2008, ce travail s'appuie sur une méthodologie qualitative. Trois techniques ont été mobilisées à cette intention : les entretiens, l'observation non participante et la technique documentaire. Les entretiens ont consisté à interroger cinq responsables d'ONG locales partenaires de l'association « La voix de l'Enfant », mais aussi douze coordinateurs formés par les ONG pour assister les SHG dans les villages de même que la majorité des membres des douze SHG correspondants. 
Dans le dernier cas, la plupart des échanges se sont déroulés sous la forme d'entretiens collectifs. Durant la phase d'observation non participante, il a été possible d'accompagner les membres de SHG dans leurs activités économiques - par exemple, la répartition, le traitement, le transport et enfin la vente des poissons pêchés par les hommes - ainsi qu'à leur domicile. Les documents collectés ont été d'ordre divers : depuis les livres de comptes des SHG jusqu' aux brochures produites par les ONG. Tous ces éléments ont été confrontés aux résultats des autres études sur le sujet.

\section{Self-Help Groups Les raisons du succès}

Bien que 1'organisation en SHG ne soit pas obligatoire, c'est pourtant à travers ce modèle que la microfinance se décline principalement en Inde ${ }^{1}$. Le fonctionnement des SHG, qui attise une curiosité grandissante de la part de la communauté des chercheurs, véhicule une dimension culturelle manifeste. D'après la Tamil Nadu Women's Development Corporation (DeW), un SHG peut être défini comme un "petit groupe, économiquement homogène, de pauvres, ruraux ou urbains, formé volontairement pour contribuer à un fonds commun destiné à être prêté aux membres selon des décisions collectives et dans le but de travailler ensemble pour le développement économique et social de leur famille et de la communauté » (Palier, 2003). Présentée comme la plus ancienne démocratie du monde, l'Inde semble donc correspondre à ce modèle ascendant au sein duquel la volonté du groupe émane d'un débat entre ses membres ${ }^{2}$. De ce fait,

1. Environ un quart des emprunteurs ont recours aux formes plus classiques du microcrédit (Ghate, 2008). 2. L'opposition avec le modèle de la Grameen Bank est apparente aussi de ce point de vue. Servet (2006) propose une comparaison des deux modèles. Jaffrelot (1998) discute du caractère présumé éternel de la démocratie indienne, du rôle du colonisateur et de l'historiographie nationaliste à ce propos. si l'essor des SHG est, en premier lieu, le signe de l'impasse des politiques traditionnelles de lutte contre la pauvreté, objet de la première section, son succès s'explique aussi parce qu'il est remarquablement adapté aux problèmes qui se posent concrètement dans les villages, analysé dans la seconde section.

\section{L'échec des politiques sociales}

Les performances de l'économie de l'Inde sont impressionnantes. Toutefois, son PIB, déjà le douzième au monde et même le quatrième en parité de pouvoir d'achat, comme son taux de croissance annuel régulièrement supérieur à $5,5 \%$ avec des pointes qui avoisinent les $10 \%$, ne peuvent occulter les disparités qui subsistent, voire s'aggravent, au sein même de sa société ${ }^{3}$. Entre État central et régions, régions riches et régions pauvres, villes et campagnes, hautes et basses castes, hommes et femmes, les clivages ne manquent pas. Tous les Indiens, loin s'en faut, ne sont pas associés à la croissance économique spectaculaire de leur pays. Les « laissés-pour-compte » du système sont tellement nombreux et démunis qu'ils rendent le contraste entre les extrêmes encore plus saisissant ${ }^{4}$. Selon les estimations, leur nombre oscille entre 400 millions et 800 millions. Il n'est peut-être pas surprenant que, sur un territoire aussi vaste (septième superficie mondiale), aussi peuplé (deuxième pays après la Chine) et dans un tel contexte historique, la mise en œuvre de politiques publiques volontaristes de réduction des inégalités n'ait pas abouti. Mais les pères fondateurs de l'Inde post-colonisation avaient pourtant affiché de fortes ambitions en la matière.

3. Les informations chiffrées de ce paragraphe proviennent du site du FMI et de la Banque mondiale.

4. Panagariya (2010) présente une explication économique de la difficulté à enclencher un cercle vertueux (secteurs intensifs en travail qualifié, en travail non qualifié, réglementations, investissements dans l'éducation supérieure...) 
Il convient de préciser que l'Inde s'est construite dans une logique de modernisation aux antipodes du projet de Gandhi, qui était ancré dans les traditions et dans lequel le « village-république » occupait une place prépondérante. Nehru souhaitait un changement fondamental rapide et qui profiterait, non pas à certaines castes, mais à l'ensemble de la population. Il conduisit ainsi son pays sur une voie où l'impulsion réformiste était communiquée par l'État central. Dans cette perspective, le regard porté sur le village devenait radicalement hostile. Ambedkar, président du comité chargé de rédiger la Constitution et ministre de la Justice de $\mathrm{Nehru}^{5}$, répondit à des élus proches du message de Gandhi et qui auraient apprécié de voir la fonction du village mieux reconnue par la Constitution :

"Qu'est-ce que le village sinon le cloaque du localisme, le repaire de l'ignorance, de l'étroitesse d'esprit et du communalisme? Je suis heureux que le projet de Constitution ait ignoré le village et adopté l'individu comme son unité de base.»

(In Jaffrelot, 1998) ${ }^{6}$

Au bout du compte, il apparaît que, si le socialisme d'État indien n'a pas atteint son objectif de réduction de la pauvreté, il a plutôt péché par excès d'ambition que par défaut. En cherchant à faire sauter des verrous, considérés comme des facteurs de blocage social, le gouvernement s'est concrètement heurté aux pouvoirs locaux. Ce qui l'a privé en retour des relais indispensables à l'exécution de sa politique de soutien aux plus défavorisés. Dans une formule peut-être simplificatrice mais qui a le mérite d'être synthétique, Daniélou (1983) n'hésite pas à affirmer que :

5. Il s'agit du même Ambedkar, qui sera vénéré des Dalits ou intouchables (Naipaul, 2002) et se trouvera à l'origine d'un mouvement de conversion au bouddhisme toujours vivace de nos jours.

6. Avec cette évocation de l'individu, la rupture est encore plus apparente avec l'ordre social traditionnel, en particulier si l'on se réfère à l'analyse « holiste » qu'en fait Dumont (1966).
«La bourgeoisie socialiste qui a pris le pouvoir en Inde s'est opposée à la hiérarchie des castes, au pouvoir modérateur des Brahmanes, prêtres lettrés, pauvres mais puissants, et à celui des princes riches mais imbus de principes chevaleresques et protecteurs du peuple. »

Les ONG ne répètent pas cette erreur aujourd'hui. Elles ont pris le parti de composer avec les panchayats (conseils de village).

Ce sont les échecs de la politique dirigiste qui ont provoqué le changement de cap de l'Inde. Dans le domaine économique, il a suffi de prendre ses distances avec les redoutables plans quinquennaux, de supprimer de nombreuses règlementations (abolition du Licence Raj en premier) et de s'ouvrir aux échanges extérieurs pour que le pays parvienne à prendre son envol. En revanche, sur les questions sociales, la situation ne s'est pas vraiment améliorée. À cet endroit, il est important de souligner que l'inégalité entre hommes et femmes n'est pas spécifique au cas de l'Inde. Il s'agit d'un mal endémique à nombre de pays en développement, cela indépendamment du modèle culturel qui les caractérise en particulier. Amartya Sen (2000) a mis au jour la problématique des «femmes manquantes »: rapport femmes/hommes inférieur à 0.95 (il dépasse souvent 1.05 dans les pays du Nord), taux de mortalité supérieur chez les femmes dans toutes les classes d'âge jusqu'à quarante ans. En faisant abstraction des cas d'infanticide, l'explication du phénomène réside prioritairement dans le moindre accès aux soins et à la nourriture de la gent féminine. Ce sont des dizaines de millions de « femmes manquantes » qui sont recensées dans les pays du Sud - en Chine et en Inde surtout ${ }^{7}$.

7. Autre illustration de ce fossé mais cette fois dans le domaine de l'éducation : en Inde, l'écart entre le taux d'alphabétisation des hommes demeurait encore largement supérieur à celui des femmes en 2007 (77\% contre $54 \%$, www.worldbank.org). 
Une politique sociale aussi ambitieuse soit-elle connaît des limites. Il ne lui est pas facile d'imposer ses exigences à l'intérieur de la famille, qui représente la sphère privée par excellente. L'inégalité entre les femmes et les hommes est pourtant criante à l'intérieur de la cellule familiale indienne. Dans le modèle traditionnel, les femmes s'occupent du foyer alors que leurs maris sont chargés d'acquérir les moyens de subsistance. Elles sont dépendantes aussi bien économiquement que culturellement parce que les Indiens sont soumis à des croyances qui perpétuent ce fonctionnement. Les hommes tirent normalement profit de cette situation. Il n'est du reste pas rare qu'ils usent de violence pour asseoir leur domination ${ }^{8}$. Dans ces circonstances, l'emprise d'eros sur polemos apparaît peu contestable. Cet état des lieux doit néanmoins être nuancé. Toutes les régions de l'Inde ne sont pas affectées de la même manière par cette réalité. Des paradoxes peuvent même être observés. Les rapports entre les hommes et les femmes sont conflictuels dans un État riche comme le Pendjab qui a subi de nombreuses invasions tout au long des siècles. Des stratégies de défense des traditions ont été développées en réaction. Le travail féminin y est mal perçu. Dans des zones plus pauvres et isolées, la relation est plus apaisée. La femme est autorisée à sortir de son enfermement pour partir travailler. Le complément de revenu qu'elle apporte à la famille est apprécié (Manier, 2006).

Ce n'est probablement pas un hasard si l'économiste du développement qui est à l'origine de « l'approche par les capabilités »(capabilities), Amartya Sen, est indien. Les droits ou libertés théoriques doivent pouvoir être distingués de ceux qui sont susceptibles d'être convertis et utilisés

8. Bénédicte Manier (2006) décrit une multitude d'exemples de violences exercées contre les femmes, fardeau dans une société « patriarcale ». La coutume du satî, crémation des veuves, en est un héritage historique. effectivement dans la vie quotidienne. Qu'une femme n'exprime pas le désir de savoir lire ne résout pas le problème que pose son analphabétisme. Elle demeure handicapée socialement sur ce point. Cela signifie simplement qu'elle vit dans un environnement qui la décourage d'envisager toute forme d'apprentissage. La pauvreté est alors définie comme une «privation de capabilités ». Et, comme les revenus ne sont pas le seul instrument à produire des capabilités ${ }^{9}$, la lutte contre la pauvreté dépasse la dimension strictement pécuniaire (Sen, 2000). Désormais, les politiques de soutien aux classes défavorisées en tiennent davantage compte, qu'elles soient menées par les États, les organisations internationales ou les ONG. Que la femme analphabète soit incitée à s'alphabétiser fait partie intégrante de leurs missions.

\section{Des formes d'actions plus adaptées}

La disparition du clivage Est-Ouest a modifié le regard porté sur les questions de développement. La vieille formule «trade, not aid» a retrouvé une seconde jeunesse au début des années quatre-vingt dix. La solution apparente consistait à devenir partie prenante du commerce international. Au niveau national, le slogan pouvait aussi être compris comme un appel à la responsabilisation, à l' « empowerment » des classes défavorisées. Pour s'en sortir, il ne fallait plus compter uniquement sur le secours de la puissance publique mais sur ses propres forces. En Inde, le programme Swarnjayanti Gram Swarozgar Yojana (SGSY) qui vise à l'auto-emploi (self employment) de franges de la population vivant au dessous du seuil de pauvreté relève de cette conception de la lutte contre l'indigence (Roesch, 2010). Le Gouvernement indien n'a pas renoncé à

9. La liste de ces « capabilités » reste à définir. Afin d'objectiver la démarche, Nussbaum (1997) propose une réflexion qui part des caractéristiques partagées par l'ensemble du genre humain, selon Aristote. 
toute intervention lourde $\mathrm{e}^{10}$ mais il a décidé de promouvoir ostensiblement les initiatives dans lesquelles les pauvres contribuent eux-mêmes à l'amélioration de leur sort. Des ONG dont l'objet est de lutter contre la pauvreté étaient présentes sur le terrain. L'État leur a délégué contre subventions une partie de ses responsabilités ${ }^{11}$. Les ONG devaient encourager la formation de SHG et les accompagner afin qu'ils soient éligibles au microcrédit auprès de banques comme la NABARD.

Dispenser des services financiers aux groupes sociaux les plus défavorisés a toujours fait partie des priorités annoncées par les différents gouvernements indiens qui se sont succédé depuis l'indépendance du pays. Pourtant, ni les politiques de nationalisation, ni la création de la NABARD en 1982 n'étaient parvenues à faire sortir les plus pauvres de l'exclusion bancaire dans laquelle ils étaient naturellement confinés. Ainsi que l'explique Palier (op. cit.), malgré un réseau particulièrement dense, le système bancaire était complètement inadapté aux besoins de ces populations : procédures complexes, calendrier déconnecté de la saisonnalité des revenus, pas de crédit à la consommation... De ce fait, les taux de remboursement étaient faibles et les banquiers assez peu enclins à accorder des

10. Le Mahatma Gandhi National Rural Employment Guarantee Act (MNREGA), qui offre une garantie légale de 100 jours de rémunération, en fait partie.

11. La finalité des ONG, auxiliaires du néolibéralisme ou modèles de solidarité, ne sera pas discutée dans le cadre de ce travail. Les critiques qui leur sont adressées reposent en grande partie sur leur reconnaissance, toute pragmatique, des structures de pouvoir existantes (Centre Tricontinental, 1998) puisque les ONG sont obligées de s'appuyer sur ces dernières pour accomplir leurs missions. De la même manière, on peut remarquer que les ONG ont des intérêts propres. En situation de rivalité avec d'autres ONG, elles cherchent à se développer et à drainer des subventions. Des stratégies « clientélistes » sont susceptibles de dériver de ces contraintes. Elles ne seront pas davantage abordées. emprunts. Ce qui provoquait immanquablement l'enlisement des plans chargés de bonnes intentions conçus par le gouvernement. C'est pourquoi, lorsque la Reserve Bank of India (RBI), l'autorité de supervision des banques, a demandé à celles-ci, en 1991, d'entreprendre des actions dans le champ de la microfinance, son changement d'optique s'est avéré total. Une année plus tard, la NABARD se lançait dans un projet pilote. Les autres institutions financières centrales lui ont emboîté le pas en s'ouvrant également dans cette direction : par exemple, Rashtriya Mahila Kosh (RMK), fonds national de crédit pour les femmes, créé en 1993 et Small Industries Development Bank of India (SIDBI) à travers sa fondation pour le microcrédit en 1999.

Les ONG sont satisfaites de la mise en place de tels programmes. Elles sont parfaitement complémentaires de ce modèle d' «empowerment » qui repose sur la microfinance. Elles y tiennent même un rôle central en faisant office d'interface entre les institutions financières et la population. Elles remplissent une fonction utile pour le prêteur aussi bien que pour l'emprunteur. Pour les banques, l'action des ONG permet déjà une baisse sensible des coûts de transaction, externalisés et pris en charge par ces dernières. Ensuite, elle offre une meilleure connaissance de la population, de ses capacités financières et de ses besoins, c'est-àdire une garantie de meilleurs taux de remboursement ${ }^{12}$. Les sommes empruntées par un individu étant souvent modiques selon les critères des banques, l'arbitrage rentabilité-coût-risque penchait rarement du côté de l'octroi de crédit. Dorénavant, les banques sont plus incitées à se prêter au jeu, en tout cas bien plus qu'avec le système des quotas de crédits accordés aux pauvres qui étaient fixés administrativement par le pouvoir central. L'information circule mieux

12. Certaines ONG, comme la Self-Employed Woman's Association (SEWA), possèdent leur propre organisme de crédit. 
au profit de tous. La présence des ONG sur le terrain, leur proximité avec la population, produisent donc d'excellents résultats, y compris du point de vue des emprunteurs.

Servet (op. cit.) atténue l'enthousiasme suscité par la microfinance depuis les années quatre-vingt dix avec comme point d'orgue le prix Nobel attribué en 2006 à Muhammad Yunus. Les limites ne manquent pas. Tout d'abord, il ne s'agit que d'une technique qui, comme toutes les techniques, doit être utilisée avec précaution ${ }^{13}$. Dans le cas contraire, les emprunteurs sont susceptibles d'être entraînés vers une spirale d'endettement qui aggravera leur situation ${ }^{14}$. Ensuite, sans même parler de rentabilité, la quête d'autonomie financière des institutions qui pratiquent le microcrédit peut les amener à sélectionner les bénéficiaires des emprunts - ce qui prive les plus nécessiteux d'accès au microcrédit. L'autre option, fixer des taux d'intérêts élevés, revient à fragiliser l'ensemble des emprunteurs. Toujours selon Servet, le nombre des organisations de micro finance qui pourraient survivre sans aide extérieure ou subvention d'aucune sorte oscille entre $1 \%$ et $10 \%$. C'est pourquoi le rôle des ONG et de l'action publique restent déterminants dans le fonctionnement des organismes de microcrédit. Leur renforcement est même primordial parce que, si le microcrédit ne constitue certainement pas le remède miraculeux qui guérira l'humanité de la pauvreté, son action peut être considérée comme positive dès lors que les attentes à son sujet sont plus modestes. Faciliter l'accès au crédit bancaire à des catégories de la population qui n'y sont pas a

13. La question de la neutralité politique de la microfinance a été posée, bien sûr. En tant que technique, l'argument est peut-être recevable mais le contexte qui a présidé à l'émergence de la microfinance, lui, ne l'est pas (Servet, 2006). Il est intéressant d'examiner le sens que Yunus donne à sa propre action (2009).

14. La crise de la microfinance en Bolivie en 2001, avec une révolte d'emprunteurs surendettés, en est une illustration. priori éligibles, les intégrer dans les circuits de la finance formelle, représente en soi une performance ${ }^{15}$.

Les avantages escomptés des SHG découlent du changement d'échelle : les SHG peuvent disposer de fonds plus importants que la simple épargne de ses membres ne l'aurait autorisé. Parallèlement, le principe de caution solidaire remplace le dépôt de garantie. L'effet de taille favorise également l'action des ONG. En se constituant en SHG, les plus pauvres bénéficient d'un meilleur suivi de la part de celles-ci. Grâce à l'accompagnement de coordinateurs locaux, les politiques systématiques de conseil, de soutien et de formation des ONG deviennent plus efficaces. La croissance rapide du nombre des SHG témoigne du jugement positif qui est porté sur eux par la population. En 2007, 41 millions d'Indiens avaient un jour obtenu un crédit bancaire par l'intermédiaire des SHG et, par rapport à l'année précédente, 9,6 millions de personnes supplémentaires décidaient d'adhérer au programme - chiffres à comparer aux 11 millions de membres de SHG en 2003. Dans un pays où approximativement $70 \%$ de la population ne possèdent pas de compte en banque ${ }^{16}$, il n'y a d'ailleurs pas que les plus démunis qui profitent de cette porte ouverte par les institutions financières. Environ $50 \%$ des membres des SHG vivent en dessous du seuil de pauvreté. Parmi les autres informations notables, plus de $90 \%$ d'entre eux sont des femmes ${ }^{17}$. En fait, les inégalités

15. Sur les obstacles rencontrés par les classes les plus défavorisées pour se lancer dans des entreprises économiques... et les moyens de les contourner, de Soto présente une analyse originale (2005).

16. Sur les disparités entre régions et même intra régionales, notamment au Tamil Nadu, voir Fouillet (2005). La demande potentielle de services de micro finance se situe dans une fourchette comprise entre 290 millions, nombre de personnes vivant sous le seuil de pauvreté, et 650 millions, nombre des personnes sans compte bancaire (Fouillet, op. cit.). 17. Ces chiffres proviennent de Ghate (2008). 
sociales s'entrecroisent et parfois se cumulent : elles existent entre familles aussi bien qu'à l'intérieur des familles. Ces données brutes suggèrent que les secondes sont mieux combattues que les premières. Il reste à vérifier si le règne de polemos s'en trouve concrètement menacé.

\section{L'action des Self-Help Groups}

La popularité des SHG ne doit pas présager de leur efficacité. Entre "fausse conscience » et aberrations de la « rationalité mimétique », les arguments pouvant justifier un engouement indu à leur propos ne manquent pas. Une réponse à cette question épineuse ne doit pas s'appuyer sur des idées reçues ou des effets de mode. De la même façon, elle doit éviter le piège qui consiste à empiler les anecdotes parce que, non seulement ce procédé ne constitue pas une preuve, mais il risque même de provoquer des biais dans l'analyse. Etudier si, effectivement, les réalisations des SHG sont à la hauteur des attentes de leurs concepteurs exige une approche méthodique. Dans un premier temps, c'est la manière dont l'idéal-type du SHG est bâti qui sera d'abord passée en revue dans la première section. En tant qu'instigateur de bonnes pratiques en matière de microfinance, la NABARD a élaboré des critères économico-financiers et sociaux qui servent de référentiel pour les SHG en gestation aussi bien que pour les ONG. Ensuite, il sera alors intéressant dans la seconde section de se demander si les SHG, tels qu'ils ont été construits, atteignent les fins qui leur ont été assignées.

\section{L'étude du dispositif}

Les trois ONG examinées au Tamil Nadu possèdent un dénominateur commun. Qu'il s'agisse d'Arunodhaya ${ }^{18}$, d'Indian Council for Children Welfare (ICCW), de Reaching The Unreached Trust (RTUT), leur objectif

18. Traduction : « Le soleil se lève ». déclaré est de protéger les enfants. Dans cette optique, chacune développe des programmes spécifiques qui peuvent renvoyer à des modes d'action susceptibles de différer. Il est cependant significatif que ces trois ONG se retrouvent une nouvelle fois sur un des moyens qu' elles emploient. En l'occurrence, toutes s'appuient sur la mise en place et l'encadrement de SHG. Il ne s'agit évidemment pas de l'unique moyen d'intervention de ces ONG mais il demeure toujours en bonne place dans leurs programmes d'action, tels qu'ils ressortent de leurs rapports d'activités, des brochures qu'elles diffusent ou de leurs sites Internet. En outre, c'est un autre trait qui a déjà été mentionné, $90 \%$ des membres de SHG sont des femmes. Ces constats ne peuvent être considérés comme anodins - la relation qui les unit pareillement.

Des raisons pratiques sont parfois évoquées pour expliquer la féminisation des SHG. Une phase d'épargne de ses membres doit toujours précéder celle de l'octroi du crédit de la part de la banque puis sa répartition à l'issue d'une décision du groupe. Or, expliquent unanimement les responsables d'ONG rencontrés, les femmes sont bien plus capables que les hommes, non seulement de dégager l'épargne préalable, mais aussi d'utiliser les fonds prêtés à bon escient, c'est-à-dire au profit de leur famille. Dans les bidonvilles comme dans les villages de pêcheurs visités, les hommes sont suspectés de gaspiller les plus petites sommes d'argent à des fins personnelles, c'est-à-dire pour boire et s'enivrer. Il se trouve que l'alcoolisme fait partie des obstacles auxquels les travailleurs sociaux se heurtent sur le terrain au quotidien. De ce problème récurrent aux représentations qui sous-tendent l'action des ONG, la transition est alors aisée : pour sauver les enfants, il est impératif de procéder à une (ré)habilitation de la famille et ce sont les femmes qui sont les meilleures garantes de cette opération. Car, sinon, comment expliquer que des ONG dédiées à l'enfance consa- 
crent tant de ressources à l'essor des SHG qui sont constitués de femmes ? ${ }^{19}$

L'argument se comprend: dans de nombreuses cultures, les femmes sont associées aux activités altruistes, gratuites ou de proximité. Guérin (2003) remarque à juste titre que «l'opposition entre mobiles intéressés et désintéressés apparaît surtout comme une reconstruction normative de la réalité ». Cela ne retire rien au fait que les femmes sont d'ordinaire jugées dépositaires de cette dimension du comportement humain ${ }^{20}$. Tout se passe comme si, à l'intérieur de la famille, la division du travail était nette : la loi, ce qui est tranché et fort, relève de l'homme tandis que l'amour, le lien, décrivent le domaine de la femme. Ainsi, quand l'homme assure son emprise sur la famille, la femme étant réduite à un état de dépendance absolue, un principe de lutte, de conflit prévaut. Le poids de polemos est écrasant. En somme, le diagnostic posé par les ONG - un rééquilibrage des rapports entre l'homme et la femme, plus équitable, est vital pour le bon fonctionnement de la cellule familiale - se traduit mécaniquement par une montée en puissance d'eros.

Pour que ce scénario dans lequel polemos s'efface devant eros se réalise, cela au profit de l'enfant, la position de la femme au sein de la structure familiale doit donc être singulièrement renforcée. Cette autonomisation graduelle est favorisée par l'action des SHG. Mais ce n'est pas sans soulever des problèmes en matière d'évaluation. Comment peut-on être sûr qu'un SHG remplit avec efficacité sa mission au service des femmes qui en sont membres ? Pour

19. Dans cette constellation de SHG féminins, les exceptions n'infirment pas la règle. A Sadayankuppam, Arunodhaya a encouragé la création de SHG masculins. Ici, il s'agit de tribus en voie de sédentarisation. L'action des ONG comprend également leur enregistrement auprès de l'état civil, signe que tout est à construire avec elles. Dans ces familles, il faut prendre autant soin de l'homme, à travers l'exercice des activités économiques, que de la femme. standardiser les pratiques, la NABARD affiche une liste de critères supposés guider les SHG dans la direction souhaitée. Ces recommandations ne peuvent être prises à la légère. Bénéficier d'un adossement bancaire en dépend tout simplement. En effet, si ces indications ne revêtent aucun caractère d'obligation légale, les banques commerciales, qui sont souvent réticentes et mal informées sur le sujet, utilisent quasiment en l'état les critères de la NABARD ${ }^{21}$. C'est pourquoi les ONG qui encadrent des SHG répercutent, via leurs coordinateurs, ce guide des bonnes pratiques au niveau de leurs propres SHG. Par essaimage, c'est peu ou prou l'ensemble des SHG qui devient ainsi normé.

L'investigation des caractéristiques synonymes de performance pour un SHG se révèle fort instructive. La NABARD précise d'emblée que l'évaluation d'un SHG diffère de celle d'un individu dans le sens où ce ne sont pas des questions de caution ou de nantissements qui doivent être pris en compte. La solidité financière n'est pas en jeu ici. L'objectif est d'appréhender la « dynamique de groupe » à travers des caractéristiques comme «la cohésion, la vigueur, le fait d'être guidé par des objectifs, la participation des membres, les décisions démocratiques et le leadership collectif $\gg$. Le point est important : pour un banquier, la meilleure garantie de remboursement est de se trouver face à un véritable groupe, uni, solidaire et qui fonctionne de façon démocratique. Le scénario à éviter est d'accorder un crédit à un assemblage artificiel de personnes qui s'est constitué

20. Autre illustration, une grande partie des expériences menées dans le cadre de l'économie solidaire concerne exclusivement les femmes (Guérin, 2003). 21. Dans son introduction sur «Les méthodes d'évaluation » des SHG, la NABARD prend elle-même en exemple « les quelques banques commerciales proactives, banques rurales régionales et banques coopératives » qui ont élaboré leurs propres critères. L'analyse des paragraphes suivants s'appuie sur l'intégralité du document (www.nabard.org). 
ad hoc. À partir de là, la NABARD s'appuie sur la définition classique des SHG (proximité géographique, homogénéité ${ }^{22}$, épargne préalable, responsables élus, décisions démocratiques...) pour faire émerger un ensemble de critères qui puissent être commodément traduits sous la forme d'une notation.

En définitive, selon la grille d'évaluation de la NABARD, quinze critères permettent d'évaluer les SHG. Une note sur 10 est attribuée à chacun d'entre eux ${ }^{23}$. La note globale d'un SHG ne peut donc excéder 150. La NABARD conseille aux banques de prêter des fonds dès que le SHG atteint un score de 120. En deçà, il est estimé trop peu solide et dynamique. Il est alors recommandé au SHG de travailler ses lacunes avant de se (re)présenter pour postuler à un crédit bancaire. Ces quinze critères peuvent être divisés en trois catégories :

- l'organisation du groupe (composition, ancienneté, fréquence des réunions) ;

- son fonctionnement (taux d'assistance aux réunions, tenue d'un compte rendu de ces réunions, participation de tous aux discussions, processus décisionnel réellement démocratique, connaissance des règles) ;

- ses caractéristiques financières (fréquence de l'épargne, mode de recouvrement de l'épargne et des prêts aux membres, décaissement des prêts, taux d'intérêt pratiqué envers les membres, ratio prêt/épargne, taux de recouvrement des

22. A propos de l' « homogénéité » (caste, sexe, activité...), la NABARD insiste sur une catégorie, celle du statut économique : une relation de dépendance, une dette à rembourser par exemple, est incompatible avec l'appartenance des personnes concernées au même SHG. Ces cas ne sont pas toujours évidents à détecter pour les coordonnateurs.

23. Pour illustration, la qualité de la « composition» d'un SHG est mesurée en fonction de son homogénéité : un groupe jugé homogène recevra une note de 10 et, dans le cas contraire, seulement 5 points lui seront attribués. prêts, tenue de livres de comptes).

Si un SHG parvient à se constituer un fonds par l'épargne de ses membres, à leur accorder des prêts et à enregistrer des taux de remboursement satisfaisants, le crédit bancaire ne fait que stimuler l'activité en mettant de l'huile dans les rouages. En faisant abstraction d'éventuels effets pervers, il joue un rôle de facilitateur. En aucune façon, le prêt de la banque ne doit être perçu comme le paramètre susceptible de rendre le SHG viable. Dans ce cas, le SHG est considéré trop fragile. En d'autres termes, la période d'essai - car c'en est une - ne s'est pas avérée concluante. Face au risque élevé, la banque refusera d'accorder un crédit au SHG. On comprend mieux la place du critère « ancienneté du SHG », évaluée positivement dès qu'elle dépasse une durée d'un an. De manière générale, l'action de standardisation de la NABARD est axée sur les critères financiers qui allouent 70 points sur les 150 . Au-delà de la pure arithmétique, c'est sa position institutionnelle, c'est-àdire sa responsabilité envers les banques commerciales, qui justifient ce tropisme.

La NABARD admet que d'autres facteurs doivent être pris en compte dans l'appréciation de la dynamique du groupe. D' ailleurs, dans ce document intitulé « Les méthodes d'évaluation », mais avant la présentation de la synthèse des quinze critères, un « progrès personnel/social » est évoqué avec « un niveau minimal d'alphabétisation devant être obtenu » et du « travail social » qui doit être accompli. Néanmoins, ces éléments ne se retrouvent pas dans le tableau final. Un autre exemple, encore plus éclatant, atteste que la NABARD reconnaît la place des critères non financiers. Dans un «Manuel sur la formation des SHG » destiné aux ONG, elle explique que la première démarche consiste à détecter des pauvres qui pourraient être intégrés aux SHG. Mais qu'est-ce qu'une famille pauvre? Sur les onze critères que la

24. Non daté, p. 5. 
NABARD mentionne alors ${ }^{24}$, deux seulement se réfèrent au niveau strictement économique (revenus, emprunt auprès d'un prêteur privé), les neuf autres possèdent une dimension sociale (source d'approvisionnement en eau, alimentation, santé, distance aux sanitaires, alphabétisation des adultes, scolarisation des enfants, alcoolisme, matériaux de l'habitation, caste). Pour la NABARD, sur le terrain, les stigmates de la pauvreté sont plus sociaux qu'économiques $^{25}$.

De leur côté, dans leur approche des SHG, les ONG mettent l'accent sur ces aspects sociaux. Elles inversent la hiérarchisation entre le financier et le social. Ainsi, dans un manuel diffusé en 2002 par ICCW ${ }^{26}$, les SHG sont décrits comme des composantes d'un « environnement créatif pour le changement social ». La dimension économico-financière ne peut être négligée : elle constitue un pilier de l'ensemble de l'édifice, une des conditions de la promotion du statut de la femme. Toutefois, ce sont les changements psychologiques, culturels, institutionnels et sociaux que les ONG valorisent en priorité. Les aspects économiques et sociaux se rencontrent, et se renforcent. Chaque catégorie d'agents économiques se focalise sur ceux qui renvoient à son champ d'intervention spécifique : les ONG remarquent l'étendue de la pauvreté et appréhendent alors le rôle des SHG dans une perspective sociale ; la NABARD, privilégie la dimension financière. Mais, quelle que soit la place respective des facteurs économiques et sociaux, leur action s'inscrit effectivement dans ce plan d'amélioration de la position de la femme dans la famille indienne ou, pour revenir à la terminologie de Sen, d'expansion de ses « capabilités ».

25. Ce même document (p. 23) propose une liste de seize critères qui doit permettre d'évaluer les SHG. Cette fois, le niveau d'éducation (lecture, écriture) est bien présent mais la finance demeure prégnante. 26. Contact : iccw.delhi@gmail.com
Selon les plus ardents défenseurs des SHG, le mouvement doit se dérouler progressivement, sans bouleverser les structures existantes. La NABARD suggère aux coordinateurs de SHG de s'adresser aux anciens qui, dans les villages, sont les gardiens de la tradition. Le but est de les rassurer pour pouvoir se reposer sur leur autorité morale. Il ressort que la mission des coordinateurs ne se réduit ni à évaluer les SHG à la manière d'une agence de notation, afin de prodiguer des conseils permettant d'améliorer leur chance de décrocher un crédit, ni à les former aux rudiments de la négociation en vue de la rencontre décisive avec le banquier ${ }^{27}$. La tâche des coordinateurs doit d'abord être comprise comme une entreprise de légitimation des SHG. C'est ainsi que ces représentants des ONG perçoivent leur action. Il faut préciser que la tentative d'ancrer les SHG dans les esprits vise non seulement les anciens et les hommes mais aussi les membres des SHG qui sont susceptibles, à l'occasion, de s'interroger sur leur efficacité. La discipline imposée, les efforts consentis, ne sont pas négligeables. L'évaluation du dispositif devient alors déterminante. Les objectifs sont-ils atteints?

\section{Des effets mal appréciés}

La mesure des retombées tant économiques que politiques se heurte à de multiples obstacles.

- Au niveau économique, les activités des membres des SHG se déroulent surtout dans le champ de l'économie informelle, qui est caractérisé par le vague et l'imprécision. De plus, elles dépendent en général du secteur primaire c'est-à-dire d'un environnement où les aléas sont nombreux (climat,

27. Les coordinateurs recommandent ainsi de ne pas s'affoler si le chargé de clientèle fait patienter l'emprunteur potentiel, a priori fragile, pendant un long moment. Cela fait partie des techniques de déstabilisation les plus courantes. 
santé du bétail...) Et c'est sans compter la présence de problèmes plus classiques d'évaluation : pertinence des indicateurs utilisés (revenu, patrimoine) ou biais statistiques (la remontée du cours de l'or, composante essentielle du patrimoine des familles, n'explique-t-elle pas en grande partie l'augmentation des richesses ?).

- En ce qui concerne l'appréciation des conséquences sociales, le choix de l'indicateur (scolarisation croissante des enfants, alphabétisation des femmes, baisse des violences conjugales, participation des femmes à la vie politique...) et la difficulté à recueillir sur le terrain des informations exactes sont également des écueils ${ }^{28}$. S'ajoute la dimension temporelle. Selon le schéma idéal, les SHG doivent libérer les femmes de leur état de soumission afin que la cellule familiale indienne s'ouvre à de nouveaux modes de fonctionnement. Dans une telle configuration, des changements de mentalité se trouvent être à l'origine de changements de comportements. Il est donc logique que ces évolutions se produisent avec une certaine lenteur. L'éventuel avènement d'eros ne peut survenir que dans la longue durée.

Ces réserves n'interdisent évidemment pas la réalisation d'études d'impact. Les plus significatives d'entre elles portent sur la microfinance dans son ensemble, tout en faisant état de différences entre ses modalités (SHG, institutions de microfinance comme la Grameen Bank, prêts individuels). Après avoir effectué un tour d'horizon de ces enquêtes, Guérin et al. (2009) en dressent un bilan mitigé. Les résultats les plus probants se situent indéniablement dans le champ

28. Exemple : ICCW (2007) recense les interventions de son centre de crise destiné à protéger les enfants. Le danger d'être confronté à un artefact statistique n'est pas mince : en effet, l'évolution des chiffres signifie-t-elle que l'existence du numéro d'urgence est mieux connue au sein de la population, que celle-ci est plus sensibilisée aux questions de violence ou que les violences elles-mêmes augmentent? économique. Globalement, $67 \%$ des sommes empruntées sont utilisées à des fins productives ( $36 \%$ pour les $\mathrm{SHG}, 82 \%$ pour le modèle Grameen ${ }^{29}$, principalement pour aider le développement de micro-entreprises familiales implantées en milieu rural ${ }^{30}$. Dans une autre étude, une hausse de $32 \%$ des créations d'entreprises est même relevée (Banerjee et al., 2009). Si elles disposent d'assez peu de marges de manœuvre pour se prémunir contre les accidents de la vie économique ou familiale, ces entreprises réussissent dans deux tiers des cas à procurer une hausse des revenus à la famille du créateur (Guérin et al., 2009).

En dépit de l'augmentation de la population en Inde, le nombre des personnes vivant sous le seuil de pauvreté, s'il est défini à un dollar par jour, a diminué en vingt cinq ans de plus de $40 \%$. En revanche, comme par un système de vases communicants, les effectifs de la classe des individus vivant avec moins de deux dollars et demi par jour se sont trouvés considérablement accrus (Banque mondiale, 2008) ${ }^{31}$. S'élever au-dessus du seuil de pauvreté ne signifie pas se rapprocher du niveau de revenus des classes moyennes urbaines. Les progrès sont donc réels mais légers. L'action des SHG participe de cette logique. Qu'il s'agisse des revenus générés par l'activité économique, de la souplesse, du plus grand confort autorisés par l'emprunt, une amélioration du bien-être des ménages

29. Dans le cas des SHG que nous avons rencontrés par l'intermédiaire de RTUT, ICCW et Arunodhaya, pas nécessairement représentatifs de ce qui se pratique sur l'ensemble du territoire, une très grande majorité de femmes s'est lancée dans des activités économiques (vente de poisson, de riz...).

30. Il arrive que l'entreprise soutenue soit celle du mari, et non celle de son épouse qui a souscrit un emprunt. Ce « détournement » du crédit bancaire est peut-être préjudiciable par rapport au projet d'émancipation de la femme par son travail mais pas en termes de révision de son statut. Sa capacité à mobiliser des fonds renforce sa position au sein du couple.

31. www.worldbank.org 
apparaît à travers l'évolution de leurs dépenses de consommation (explosion des postes santé et éducation, ce qui établit d'ailleurs un lien avec les effets sociaux, à travers une modification des coefficients budgétaires). Dans le même ordre d'idée, une plus grande attention est accordée au durable : les habitations se sont cimentées (Guérin et al., op. cit.)

En parallèle, bien que la hausse de la valeur de l'or exige une certaine prudence dans son analyse, le ratio endettement/patrimoine a tendance à baisser pour $90 \%$ des ménages. Mais c'est pourtant sur les personnes qui voient leur situation se détériorer et qui accumulent alors crédit sur crédit que les médias braquent leurs projecteurs $^{32}$. Il est vrai que les évènements ne suivent pas forcément le cours espéré dans le sens où le microcrédit ne se substitue pas entièrement aux prêteurs privés. En fait, les ménages procèdent plutôt à une diversification de leurs sources de financement. Malgré leurs taux élevés, les prêteurs privés offrent davantage de flexibilité aux ménages que le microcrédit. De surcroît, ce sont souvent des personnages importants dans les villages et il serait imprudent de couper les liens avec eux. C'est pourquoi, tout en voyant leur part décliner, les prêts des moneylenders représentent encore $30 \%$ de l'endettement des ménages ${ }^{33}$. La multiplicité des accès à un financement, associée à la baisse du coût du crédit, facilite la gestion de trésorerie de la grande majorité des ménages - ce qui est une avancée sensible - mais elle fragilise en même temps les plus vulnérables (Guérin et al., op. cit.)

La belle mécanique du microcrédit est

32. Pour illustration, le reportage de Stéphanie Lebrun et Philippe Levasseur sur France 2 : «Banquier des pauvres » (Envoyé Spécial, 14 mai 2009).

33. De par leur faculté d'obtention des crédits bancaires, les femmes membres de SHG bénéficient d'une bonne réputation auprès des prêteurs privés. Ce qui alimente leur endettement auprès de ces derniers. susceptible de s'enrayer parce qu'une dette préalable existait avant que le crédit ne soit accordé. Ce cas de figure est susceptible de se produire si un proche parent de membre de SHG n'a pas remboursé un prêt individuel qui lui avait été accordé antérieurement. La banque peut alors exiger que le SHG utilise l'argent qui lui est prêté pour rembourser l'ancien emprunt (Kalpana, 2008). L'endettement du groupe de femmes se substitue à celui d'un homme. Dans ces conditions, l'objectif d'autonomisation des femmes s'éloigne. Ce problème de dette originelle qui vient parasiter le dispositif ne concerne pas uniquement la relation envers les banques. Plusieurs coordinateurs nous ont indiqué qu'ils avaient parfois observé une dépendance initiale entre des membres d'un SHG, ou entre leurs familles. Cette inégalité de départ produit des résultats indésirables : les poissons les plus chers à la revente ou les ressources financières reviennent aux « créanciers ». La capacité des coordinateurs à rééquilibrer le fonctionnement du SHG est extrêmement limitée. Ils jugent ce phénomène rare. Mais l'édifice reste fragile et les risques de détournement existent.

De leur côté, la plupart des indicateurs sociaux ne dévoilent aucune inflexion véritable à ce jour. Avant d'être annonciatrice d'un changement, la croissance des dépenses de santé et d'éducation, soulignée plus haut, doit d'abord être comprise comme un décollage à partir du point le plus bas. Les violences familiales ne connaissent pas non plus de régression fulgurante. Pourtant, des signes encourageants sont également apparus. Les rencontres régulières au sein des SHG ont amorcé un processus de prise de conscience de la part de leurs membres. Savoir que d'autres femmes partagent les mêmes soucis et découvrir qu'il est possible de se comporter autrement constitue une condition nécessaire, quoique non suffisante, à une révolution sociale même silencieuse. Palier (op . cit.) se réfère à une étude attestant, grâce aux SHG, d'un saut en 
matière de confiance en soi (de $20 \%$, avant la naissance du SHG, à 89 \%, après !), de refus des violences domestiques, ainsi que d'un recul significatif de l'adoption d'attitudes résignées face à des difficultés ${ }^{34}$. Dans ces circonstances, l'espoir est permis.

À l'échelle du pays, l'indicateur de la participation des femmes (IPF) ${ }^{35}$ a progressé : entre 1996 et 2006, il est passé de 0.413 à 0.451 (PNUD, 2009) $)^{36}$. Cela ne démontre rien pour les SHG eux-mêmes mais il est difficile d'imaginer que ce sentiment de posséder des droits, ce courage insufflé par l'action du groupe ne les a pas poussés dans la bonne direction. Les femmes membres de SHG sont moins recroquevillées sur leurs problèmes. Elles s'ouvrent sur l'extérieur à tous les niveaux, le moindre n'étant pas celui de la mobilité spatiale (Guérin et al., op. cit.) Pour conduire leurs projets, les femmes sortent en effet de leur domicile et élargissent leur périmètre d'action. Les hommes ne sont pas dupes du risque de perte de pouvoir qu'ils encourent. Selon les équipes d'ONG, ils n'hésitent alors pas à reprocher à leurs femmes de négliger leurs obligations familiales. Que polemos cherche parfois à résister n'est pas forcément une mauvaise nouvelle : c'est plutôt le signe que les lignes bougent.

Des effets pervers existent : des femmes tellement investies dans leur micro-entreprise et convaincues qu'il s'agit d'une chance exceptionnelle à saisir pour elles et leur famille sont susceptibles de retirer leurs enfants de l'école pour que ceux-ci les assistent dans leur activités ${ }^{37}$. Toutefois, il n'est pas exagéré de supposer que les SHG fini-

\footnotetext{
34. Le bien-être que les membres des SHG affichent, lorsqu'ils sont interrogés sur leur intérêt pour les sujets de discussion non économiques abordés durant leurs réunions (droit des femmes, école...), est frappant.

35. La dénomination de l'IPF en anglais est le GEM (Gender Empowerment Measure).

36. www.undp.org

37. Ce danger a été mentionné à deux reprises lors d'entretiens avec des responsables d'ONG.
}

ront par renforcer la scolarisation. Pour que les enfants ne subissent pas les mêmes tourments que les mères, l'éducation est présentée dans le groupe comme la meilleure issue possible. Il faut savoir que les campagnes traditionnelles d'information sur l'école sont loin de pouvoir se targuer de performances exceptionnelles. L'action des comités d'éducation des villages (VEC), qui sont composés de notables, de parents et d'enseignants, ne débouche pas sur des effets significatifs (Banerjee et al., 2007). Les «bonnes idées » ne font pas « les bons comportements ». Or, en se reportant aux expériences de Kurt Lewin sur la dynamique de groupe (1958), un SHG peut être assimilé à une instance dans laquelle les femmes prennent la parole et s'engagent devant les autres membres, y compris sur les questions d'éducation quand elles sont débattues. Il s'agit bien des conditions optimales pour obtenir un passage à l'acte.

À la réflexion, certains des reproches adressés aux SHG, et plus généralement aux avatars de l'émancipation de la femme indienne, sont en fait le témoignage d'une évolution favorable. Prêter uniquement une oreille attentive aux demandes de la population féminine à l'approche des élections, parce que l'on estime que les SHG sont un « réservoir de voix », est décevant si l'on se focalise sur les promesses qui ne sont pas tenues. Mais cela signifie également que les femmes existent politiquement, qu'elles sont incontournables et que les candidats doivent désormais traiter de leurs préoccupations. Ce qui n'était pas le cas auparavant. Si la route est encore longue, des étapes ont été indiscutablement franchies. Pande (2000) raconte qu'une élue s'est plainte devant le chef du gouvernement du Madhya Pradesh de l'absence de chaises pour les femmes invitées à une réunion. Elle a reçu des excuses. Quelques décennies plus tôt, elle aurait été pendue pour son audace... Les responsables politiques sont peut-être plus respectueux des femmes que les maris car ils sont exposés à une éventuelle action 
collective. Mais, à tous les niveaux, les SHG semblent aider leurs membres à grignoter tout doucement un terrain inoccupé durant des siècles.

\section{Conclusion}

Les observations de notre enquête terrain menée au Tamil Nadu sont corroborées par les travaux de référence sur la microfinance en Inde (Guérin et al., 2009) A ce stade, le bilan des SHG peut être considéré comme encourageant. Ni les risques d'altération du dispositif mis en place, ni les résistances à ce processus d'émancipation ne doivent être sous-estimés. Mais, si l'on accepte l'idée que ces associations ne peuvent constituer une solution miraculeuse aux problèmes rencontrés par les femmes dans les familles les plus pauvres, plusieurs éléments positifs ressortent dans l'économique comme dans le social. Dans ce dernier domaine, quoique les performances ne soient véritablement appréciables que dans la longue période, la prise de conscience des femmes que la soumission aux hommes n'est pas inéluctable, leur sentiment que la voie vers une amélioration de leur sort est toute tracée, sont porteurs à terme au moins d'un immense espoir. Pour les ONG, un rééquilibrage graduel entre les principes de liaison, eros, et de conflit, polemos, ne relève pas de l'utopie. Elles se positionnent dans le long terme.

Â ce propos, il faut savoir qu'une intervention extérieure bien ciblée représente un formidable accélérateur. L'installation du séchoir solaire à Samiarpettai, dans le district de Cuddalore, peut ainsi être assimilée à un bouleversement en termes organisationnels. Auparavant, les femmes devaient se relayer pendant une journée entière. Désormais, elles récupèrent le poisson séché qu'elles vont vendre après une attente de seulement quatre heures. Mesuré en temps économisé pour la famille, les activités économiques et la communauté dans son ensemble, le gain est exceptionnel. Dans ce cas précis, c'est une ONG qui est à l'origine de l'intervention extérieure mais celle de l'État est tous aussi envisageable. Certes, la microfinance doit en partie son développement au libéralisme triomphant des années quatre-vingt-dix. Mais la microfinance ne prétend pas que la responsabilisation des pauvres va de pair avec une déresponsabilisation de la puissance publique. Il n'existe aucune raison objective pour que l'empowerment des femmes par le biais des SHG soit le corollaire du disempowerment de l'État. Les éventuels accomplissements des SHG n'exonèrent pas l'État indien de ses obligations envers ses citoyens. La microfinance n'est qu'un outil. Rien ne serait plus préjudiciable à la discussion que d'opposer stérilement le triptyque «promotion des SHG - déresponsabilisation de l'État promotion du libéralisme » à son contraire « critique des SHG - responsabilisation de l'État - critique du libéralisme ».

\section{L'auteur remercie :}

- L'Institut des sciences techniques et économie commerciales (ISTEC), la Voix de I'Enfant (Jérôme Baptiste) et les Centres Leclerc pour l'organisation du voyage ;

- Rémi Jardat, enseignant-chercheur, Julie Castanié et Adrien Pujol, étudiants, tous de I'ISTEC, pour le travail d'équipe dans la collecte des données ;

- les dirigeants de RTUT, ICCW et Arunodhaya, les coordinateurs et les membres de Self-Help Groups pour le temps précieux qu'ils nous ont consacré. 


\section{RÉFÉRENCES BIBLIOGRAPHIQUES}

Arunodhaya (2007). Annual Report 20062007. Arunodhaya Center for Street and Working Children.

Banerjee A., Banerji R., Duflo E., Glennerster R., Keniston D., Khemani S., Shotland M. (2007). Can Informational Campaigns Raise Awareness and Local Participation in Primary Education. Economic and Political Weekly, 14-20 avril, p. 1365-1372.

Banerjee A., Duflo E., Glennerster R., Cynthia K. (2009). The Microfinance Miracle? Evidence from a Randomized Evaluation. Working paper, mai, p. 1-26.

Centre Tricontinental (1998). Les ONG : instruments du néolibéralisme ou initiatives populaires? Paris, L'Harmattan.

Daniélou A. (1983). Histoire de l'Inde. Fayard.

(de) Soto H. (2005). Le mystère du capital. Flammarion.

Dumont L. (1966). Homo hierarchicus. Le système des castes et ses implications. Gallimard.

Fouillet C. (2005). La microfinance en Inde : données chiffrées et zoom sur les SelfHelp-Groups. Gret-Cirad, Espace Finance, Bulletin d'information du mardi, 6 décembre, p. 1-10.

Ghate P. (2008). Microfinance in India: A State of the Sector Report 2007, Sage.

Guérin I. (2003)Femmes et économie solidaire. Paris, La Découverte.

Guérin I., Palier J. Prevost B. (2009). Femmes et microfinance; espoirs et désillusion de l'expérience indienne. Archives Contemporaines.

Jaffrelot C. (1998). La démocratie en Inde. Religion, caste et politique. Fayard.

Jaffrelot C. (2006). L'Inde contemporaine de 1950 à nos jours. Fayard.

Kalpana K. (2008). The Vulnerability of "Self-Help": Women and Microfinance in South India, Working Paper, Institute of Development Studies, avril, p. 1-44.
Lebrun S. Levasseur P. (2009). Banquier des pauvres. Envoyé Spécial, France 2, 14 mai. Lewin K. (1958). Group Decision and Social Change. In Hartley E., Maccoby E. Newcomb T. (ed.), «Reading in social psychology », Holt Rinehart and Winston, p. 197-211.

Manier B. (2006). Quand les filles auront disparu. L'élimination des filles en Inde et en Asie. Paris, La Découverte.

NABARD (National Bank for Agriculture and Rural Development) : www.nabard.org NABARD (1992). Some Do's and Don't in linking banks and Self-Help Groups.

NABARD (non daté). A Handbook on Forming Self-Help Groups.

Naipaul V. (2002). L'Inde. Plon.

Nussbaum M. (1997). Non Relative Virtues: An Aristotelian Approach. In Nussbaum M., Sen A. (ed.), « The Quality of Life », Clarendon Press, p. 242-269.

Palier J. (2003). La collaboration entre les banques commerciales, organisations non gouvernementales et self-help groups. Quelques pratiques de microfinance indienne. In Najim A., Hofmann E. Kamala M.-G. (éd.), « Les entreprises face aux enjeux du développement durable ». Éléments d'un débat, Karthala, p. 225-252.

Panagariya A. (2010). India: The Emerging Giant. Oxford University Press.

Pande M. (2000). Le laboratoire des panchayats. Le courrier de l'UNESCO, juin, p. 32-33.

Roesch M. (2010). Un microcrédit pour acheter une vache : ... et le miracle n'aura pas lieu. Histoire d'une innovation en Inde du Sud. ISDA 2010, Montpellier 28-30 juin, p. 1-15.

Sen A. (2000). Un nouveau modèle économique. Développement, justice, liberté. Odile Jacob.

Servet J-M. (2006). Banquiers aux pieds nus. La microfinance. Odile Jacob.

Yunus M. (2009). Vers un nouveau capitalisme. Lgf. 